\title{
Upfront Treatment
}

National Cancer Institute

\section{Source}

National Cancer Institute. Upfront Treatment. NCI Thesaurus. Code C155925.

The initial treatment for a disease. 